\title{
COMMENTARY
}

\section{The lung and the brain: a dangerous cross-talk}

\author{
Paolo Pelosi*1 and Patricia RM Rocco ${ }^{2}$ \\ See related research by Quilez et al., http://ccforum.com/content/15/3/R124
}

\begin{abstract}
Brain or lung injury or both are frequent causes of admission to intensive care units and are associated with high morbidity and mortality rates. Mechanical ventilation, which is commonly used in the management of these critically ill patients, can induce an inflammatory response, which may be involved in distal organ failure. Thus, there may be a complex crosstalk between the lungs and other organs, including the brain. Interestingly, survivors from acute lung injury/ acute respiratory distress syndrome frequently have some cognitive deterioration at hospital discharge. Such neurologic dysfunction might be a secondary marker of injury and the neuroanatomical substrate for downstream impairment of other organs. Brainlung interactions have received little attention in the literature, but recent evidence suggests that both the lungs and brain can promote inflammation through common mediators. The present commentary discusses the main physiological issues related to brain-lung interactions.
\end{abstract}

The study by Quilez and colleagues [1] in this issue of Critical Care reports morpho-functional and biochemical effects of mechanical ventilation with lower $(8 \mathrm{~mL} / \mathrm{kg})$ and higher $(30 \mathrm{~mL} / \mathrm{kg})$ tidal volume $\left(\mathrm{V}_{\mathrm{T}}\right)$ on lung and brain in healthy rats. Mechanical ventilation may have a serious impact on lung structure and function, leading to ventilator-associated lung injury (VALI) as well as promoting damage of peripheral organs, including the brain. In this respect, mechanical ventilation and sedation in healthy and diseased lungs have been reported to be associated with neurologic impairment, memory, and cognitive dysfunction [2]. On the other hand, brain injury may enhance lung damage in experimental settings [3],

*Correspondence: ppelosi@hotmail.com

'Department of Surgical Sciences and Integrated Diagnostics, University of Genoa, Largo Rosanna Benzi 8, 16132, Genoa, Italy

Full list of author information is available at the end of the article probably by promoting a higher rate of pulmonary complications as well as by altering neurologic outcome [4]. Overall, the information in regard to the multiplepathway cross-talk between the brain and lungs is quite limited [5].

In the study by Quilez and colleagues [1], different variables, including lung function, plasma, and lung levels of cytokines as well as $c$-fos gene, were measured. $c-f o s$ is a marker of neuronal activation and is correlated with an increase in functional and metabolic activity in the brain, involved in the phenomena of neuronal plasticity, expressed in response to a wide range of stimuli, and implicated in processes such as gene transcription, apoptosis, or proliferation [6]. The main results of the study by Quilez and colleagues can be summarized as follows: (a) independently of higher or lower $\mathrm{V}_{\mathrm{T}}$, mechanical ventilation 'per se' (compared with spontaneous breathing) induced neutrophil infiltration, increased lung damage, and was associated with a greater release of inflammatory markers in both the lung and plasma as well as c-fos activation in central amygdala, hippocampus, paraventricular hypothalamic nuclei, and supraoptic nucleus; and (b) higher $\mathrm{V}_{\mathrm{T}}$ increased $c$-fos in the retrosplenial cortex and hypotalamus and increased tumor necrosis factor-alpha in the plasma. Therefore, mechanical ventilation promoted brain activation and the intensity of the response was increased with higher $\mathrm{V}_{\mathrm{T}}$, suggesting a cross-talk between the brain and lungs.

Different mechanisms may explain the possible negative effects of mechanical ventilation on the specific anatomical areas of the brain, even in the absence of previous lung disease [7]: (a) release of local pulmonary inflammatory mediators in the bloodstream with activation of brain response and (b) altered regional cerebral perfusion due to increased mean airway pressure, reduced lymphatic drainage, and activation of the autonomic system (Figure 1).

Brain injury-associated pulmonary dysfunction has long been attributed only to a greatly increased sympathetic activity with pulmonary venoconstriction and higher capillary permeability, but a recent study reported the role of systemic inflammatory response with pulmonary infiltration of neutrophils, cytokine release, and endothelial dysfunction triggered by an initial sympathetic 


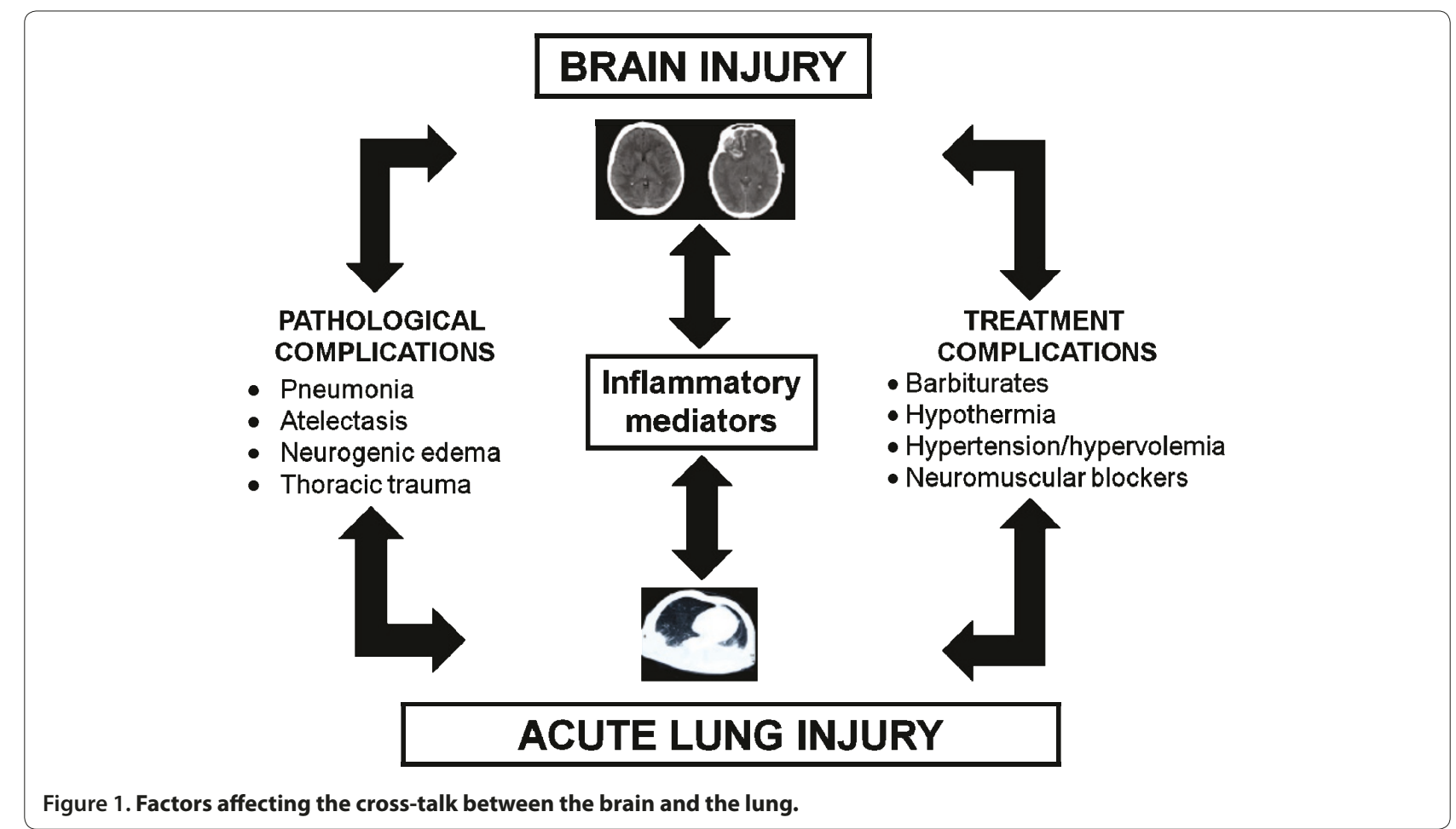

discharge [8]. A clinical study has shown that, after severe brain injury, higher $\mathrm{V}_{\mathrm{T}}$ was associated with increased risk of acute lung injury (ALI) [9]. On the other hand, ALI itself induces a systemic inflammatory response with elevated cytokines and neutrophils as well as a dysfunction of other organs, including the brain [10]. Protective ventilation could be associated not only with less VALI but also with better cerebral oxygenation and perfusion [11]. Heuer and colleagues [3] found that acute intracranial hypertension increased extravascular lung water and increased the amount of poorly aerated tissue in previously healthy lungs without alterations in gas exchange. In experimental ALI, intracranial hypertension worsened pre-existing lung damage and further increased brain edema. Conversely, ALI alone increased the circulating concentrations of the neuronal damage markers (neuronal serum enolase and S100B), but the most severe hippocampal damage was seen in animals with combined ALI and intracranial hypertension. This is in agreement with clinical reports $[11,12]$ and could indicate a reciprocal, synergistic effect of the two conditions.

The study by Quilez and colleagues, despite generating interesting data for the near future, has some limitations. First, small animals were used and thus similar results may not be obtained in larger animals with ALI. Second, rats were ventilated with very high $\mathrm{V}_{\mathrm{T}}(30 \mathrm{~mL} / \mathrm{kg}$, which is equivalent to $2,100 \mathrm{~mL}$ in average-sized adult humans)
[13]. Third, protective mechanical ventilation was not applied since no positive end-expiratory pressure (PEEP) was used. Therefore, the increase in brain damage and inflammation, even with lower $\mathrm{V}_{\mathrm{T}}$, could have been avoided by using moderate PEEP levels. Fourth, moderate hypercapnia and low pHa in the low $\mathrm{V}_{\mathrm{T}}$ group may have affected the results. Also, the duration of the experiment was quite short (3 hours), so that different effects might be observed with longer periods of mechanical ventilation. Lastly, the type of sedation during spontaneous breathing and paralyzing agents during mechanical ventilation may affect lung inflammation $[14,15]$.

In conclusion, recent data from experimental and clinical studies have shown (a) increased evidence of a cross-talk between the brain and lungs and (b) a reduction in VALI as well as brain injury when protective ventilation is adopted. Thus, early detection of VALI, through clinical signs and measurements of extravascular lung water and of brain injury by measuring specific markers, could help to optimize ventilatory settings and even the overall clinical management of critically ill patients.

Abbreviations

ALI, acute lung injury; PEEP, positive end-expiratory pressure; VALI, ventilatorassociated lung injury; $V_{T^{\prime}}$ tidal volume.

Competing interests

The authors declare that they have no competing interests. 


\section{Acknowledgments}

This commentary received financial support from the Centers of Excellence Program (PRONEX-FAPERJ), the Brazilian Council for Scientific and Technological Development (CNPq), the Carlos Chagas Filho Rio de Janeiro State Research Supporting Foundation (FAPERJ), and Coordination for the Improvement of Higher Level Personnel (CAPES).

\section{Author details}

'Department of Surgical Sciences and Integrated Diagnostics, University of Genoa; Largo Rosanna Benzi 8, 16132, Genoa, Italy. 'Laboratory of Pulmonary Investigation, Carlos Chagas Filho Biophysics Institute, Federal University of Rio de Janeiro; Av. Carlos Chagas Filho, s/n, Rio de Janeiro, 21949-902, Brazil.

Published: 30 June 2011

\section{References}

1. Quilez ME, Fuster G, Villar J, Flores C, Martí-Sistac O, Blanch L, López-Aguilar J: Injurious mechanical ventilation affects neuronal activation in ventilated rats. Crit Care 2011, 15:R124.

2. Morandi A, Brummel NE, Ely EW: Sedation, delirium and mechanical ventilation: the 'ABCDE' approach. Curr Opin Crit Care 2011, 17:43-49.

3. Heuer JF, Pelosi P, Hermann P, Perske C, Crozier TA, Brück W, Quintel M: Acute effects of intracranial hypertension and ARDS on pulmonary and neuronal damage. A randomized experimental study in pigs. Intensive Care Med 2011 May 5 [Epub ahead of print].

4. Pelosi P, Ferguson ND, Frutos-Vivar F, Anzueto A, Putensen C, Raymondos K, Apezteguia C, Desmery P, Hurtado J, Abroug F, Elizalde J, Tomicic V, Cakar N, Gonzalez M, Arabi Y, Moreno R, Esteban A; for the Ventila Study Group: Management and outcome of mechanically ventilated neurological patients. Crit Care Med 2011, 39:1482-1492

5. Gonzalvo R, Martí-Sistac O, Blanch L, López-Aguilar J: Bench-to-bedside review: brain-lung interaction in the critically ill: a pending issue revisited. Crit Care 2007, 11:216.

6. Sanz O, Estrada A, Ferrer I, Planas AM: Differential cellular distribution and dynamics of HSP70, cyclooxygenase-2, and c-Fos in the rat brain after transient focal ischemia or kainic acid. Neuroscience 1997, 80:221-232.

7. Pelosi $P$, Severgnini $P$, Chiaranda M: An integrated approach to prevent and treat respiratory failure in brain-injured patients. Curr Opin Crit Care 2005 11:37-42.

8. Mascia L: Acute lung injury in patients with severe brain injury: a double hit model. Neurocrit Care 2009, 11:417-426.

9. Mascia L, Zavala E, Bosma K, Pasero D, Decaroli D, Andrews P, Isnardi D, Davi A, Arguis MJ, Berardino M, Ducati A; Brain IT group: High tidal volume is associated with the development of acute lung injury after severe brain injury: an international observational study. Crit Care Med 2007, 35:1815-1820.

10. Bickenbach J, Zoremba N, Fries M, Dembinski R, Doering R, Ogawa E, Rossaint $R$, Kuhlen R: Low tidal volume ventilation in a porcine model of acute lung injury improves cerebral tissue oxygenation. Anesth Analg 2009, 109:847-855.

11. Holland MC, Mackersie RC, Morabito D, Campbell AR, Kivett VA, Patel R, Erickson VR, Pittet JF: The development of acute lung injury is associated with worse neurologic outcome in patients with severe traumatic brain injury. J Trauma 2003, 55:106-111.

12. Hopkins RO, Gale SD, Weaver LK: Brain atrophy and cognitive impairment in survivors of acute respiratory distress syndrome. Brain Inj 2006, 20:263-271.

13. Protti A, Cressoni M, Santini A, Langer T, Mietto C, Febres D, Chierichetti M, Coppola S, Conte G, Gatti S, Leopardi O, Masson S, Lombardi L, Lazzerini M, Rampoldi $E$, Cadringher $P$, Gattinoni L: Lung stress and strain during mechanical ventilation: any safe threshold? Am J Respir Crit Care Med 2011, 183:1354-1362.

14. Hughes CG, Pandharipande PP: Review articles: the effects of perioperative and intensive care unit sedation on brain organ dysfunction. Anesth Analg 2011, 112:1212-1217.

15. Moriondo A, Marcozzi C, Bianchin F, Passi A, Boschetti F, Lattanzio S, Severgnini P, Pelosi P, Negrini D: Impact of respiratory pattern on lung mechanics and interstitial proteoglycans in spontaneously breathing anaesthetized healthy rats. Acta Physiol (Oxf) 2011 Apr 22 [Epub ahead of print]

doi:10.1186/cc10259

Cite this article as: Pelosi P, Rocco PRM: The lung and the brain: a dangerous cross-talk. Critical Care 2011, 15:168 